\title{
Komplementärmedizin - subtiler Materialismus? Oder: Die Frage nach dem Bewusstsein
}

Thure von Uexküll, einer der bedeutenden deutschen Psychosomatiker, hat wiederholt darauf hingewiesen, dass die Medizin spätestens seit Virchow und im Gefolge von Descartes der impliziten Metapher gefolgt ist, der menschliche Organismus sei eine Maschine [4]. Kompliziert zwar, aber im Prinzip den Regeln der Kausalität und Lokalität gehorchend. Das bedeutet, dass Wirkungen, wie etwa die von pathogenen Noxen oder pharmakologischen Substanzen, durch unmittelbare räumliche Nähe und Signalübertragung zustande kommen und im Körper ebenso weitervermittelt werden. Aus diesem Grund war die Entdeckung des synaptischen Spalts eine Katastrophe, weil dieser die Kontinuität der elektrischen Reizleitung zu verhindern schien, und die Entdeckung der Neurotransmitter ein Triumph, weil dadurch die implizite Grundannahme einer kontinuierlichen, materiell und lokal vermittelten Kausalität aufrechterhalten werden konnte. Dieses Paradigma hat der Medizin grosse Erfolge beschert, vor allem bei der Entdeckung, auf welche Art Krankheiten zustande kommen und wie sie wieder heilbar sind. Auch wenn zwischen den Erkenntnissen von Pathologie und Pathophysiologie und den Möglichkeiten einer sich daraus ergebenden Therapeutik oftmals grosse Lücken klaffen, muss jeder unvoreingenommene Beobachter zugeben, dass dieses Maschinenmodell des Organismus vorderhand zu grossartigen Einsichten und beachtlichen therapeutischen Möglichkeiten geführt hat.

Es sind vor allem die chronischen, die rekurrenten, die idiopathischen und komplexen Krankheiten, bei denen dieses Paradigma auf seine Unzulänglichkeit oder Beschränktheit verwiesen wird und bei denen die Komplementärmedizin in vielerlei Spielarten ins Bild kommt. Die immer wiederkehrende kindliche Tonsillitis oder Otitis media, zum Beispiel, lässt sich auf dem Hintergrund des Maschinenparadigmas und der lokalen Kausalität gut als eine Invasion von pathogenen Keimen in den Organismus verstehen und durch eine antibiotische Therapie behandeln. Auch das Wiederkehren lässt sich ent- weder als eine Reinvasion von resistenten Keimen, die sich früheren antibiotischen Behandlungen entziehen konnten, oder als systemische Schwächung der Abwehrlage verstehen. Aber die Behandlung eben dieser systemischen Schwäche oder die Durchbrechung des Teufelskreises von antibiotischer Behandlung und Resistenz durch noch potentere Antibiotika ist auf dem Hintergrund des gleichen Paradigmas kaum vorstellbar. Bakterien - und vermutlich auch Viren - sind allen gegen sie gerichteten Massnahmen immer einen Schritt voraus, allein schon durch die Geschwindigkeit ihrer Teilungszyklen und der simplen Wahrscheinlichkeit einer einzigen genetischen Mutation, die Resistenz gegenüber der antibiotischen Massnahme verschafft, abgesehen von anderen Schutzmechanismen. Ein simpler mechanistischer Eingriff zur Behebung des Problems, in der Manier eines Auto- oder Flugzeugmechanikers, führt dann zu immer weiter- und tiefergehenden Störungen. Die Homöopathie und andere komplementärmedizinische Disziplinen haben diesen Zusammenhang früh erkannt und behandeln ihn unter dem Stichwort der «Unterdrückung». Modern gesprochen verbirgt sich dahinter ein Eingriff in die komplexen Selbstregulationsmechanismen des Organismus. Und damit ist eine neue Leitmetapher angesprochen, die sich nur allmählich innerhalb der modernen Medizin Raum verschafft, die aber besser geeignet ist, eine Brücke zu den Modellen der Komplementärmedizin zu schlagen.

Dies ist die Metapher eines komplexen, sich selbst regulierenden Systems. Ausgehend von den Gedanken Ludwig von Bertalanffys [1], der schon früh darauf verwies, dass Lebewesen keine Automaten, sondern Systeme mit autonomen Eigenschaften sind, hat die Systemtheorie sich einerseits selbst weiterentwickelt und andererseits neue Bereiche für sich entdeckt. Die Weiterentwicklung hat dazu geführt, dass man feststellte, dass viele Phänomene sich verstehen lassen als Ausdruck der Selbstregulation eines weitgehend selbständigen, eigenen Gesetzen gehorchenden und seine eigenen Strukturen 
produzierenden Systems [3, 5]. Die Entdeckung neuer Bereiche hat kürzlich dazu geführt, dass man dieses Modell auch auf Krankheit und Therapie angewandt hat. Man kann etwa, wie Michael Hyland dies vorschlägt, den Organismus als komplexes, sich selbst regulierendes System verstehen, der Gesundheit als Ausdruck der vielfältigen balancierten Interaktionen seiner Teilsysteme selbst produziert [2], ein Gedanke, der sehr nahe an von Uexkülls Wort von der Gesundheit als aktiver Leistung des Organismus herankommt. Deshalb gibt es unterschiedliche Störungen dieser Aktivitäten des Organismus. Auf einer sehr einfachen Ebene, dem der akuten Krankheit, wird ein Teilsystem durch einen lokalen, kausalen Einfluss in seiner Integrität gestört. Wenn man etwa mit Salmonellen verseuchtes Tiramisu in ausreichenden Mengen isst, dann wird selbst der robusteste Mensch die einen oder anderen Symptome zeigen. Dies ist die erste Ebene oder Störung erster Ordnung. An dieser Stelle sind konventionelle medizinische Massnahmen meistens am erfolgreichsten, die auf einem rein mechanistischen Verständnis der pathologischen Prozesse beruhen. Wenn es aber zu häufigen Störungen verschiedener oder der immer gleichen Teilsysteme kommt oder wenn therapeutische Massnahmen zu stark in die Selbstregulationsprozesse des Körpers eingreifen, dann kann es zu einer Störung zweiter Ordnung oder zu einem Netzwerkfehler kommen. Dann werden nämlich die inneren Richtwerte selbst verändert, und der Organismus reproduziert statt Gesundheit immer wieder Krankheit, weil er nämlich den inneren Zielwert verändert, der anzustreben ist. Dies wäre bei allen chronisch-entzündlichen, immunologischen oder metabolischen Störungen der Fall und möglicherweise auch bei vielen kanzerogenen Prozessen. Ein Paradebeispiel für eine solche komplexe Netzwerkstörung oder Störung zweiter Ordnung wäre etwa das chronische Müdigkeitssyndrom. Viele Einzelereignisse unterschiedlicher Natur können am Schluss zur selben gemeinsamen Endstrecke der typischen Symptome von Erschöpfung, Unfähigkeit zur Erholung, immunologischen Auffälligkeiten, Infektanfälligkeit, lokalen Entzündungsreaktionen führen. Im einen Fall sind wiederholte persistierende Infekte oder schlecht ausgeheilte und verschleppte Grippen zusammen mit übermässiger Belastung die Auslöser. Im anderen Falle vielleicht eine idiopathische Unverträglichkeit von chemischen Substanzen und Nahrungsmitteln oder Nahrungsmittelzusatzstoffen. Im einem weiteren Falle vielleicht eine unverarbeitete biographische Situation, zu der ein psychischer oder immunologischer Belastungsfaktor hinzutritt. Das Endresultat ist die Tatsache, dass es dem Organismus nicht mehr gelingt, die ursprüngliche harmonische und balancierte Ausgangslage selbst wieder herzustellen, die wir Gesundheit nennen, weil er seinen inneren Richtwert verändert hat und selbsttätig und irregeleitet Krankheit erzeugt.

Diese letztgenannten Formen von Krankheiten, oder Krankheiten zweiter Ordnung, von Michael Hyland als Netzwerkfehler bezeichnet, sind die eigentliche Domäne der verschiedenen Naturheilverfahren und der Komplementärmedizin.
Ihnen allen gemeinsam ist, dass sie versuchen, die fehlgeleitete oder fehlende Regulationsfähigkeit wiederherzustellen, indem sie dem Organismus Impulse geben, auf die er wiederum reagiert. Es ist wichtig zu betonen, dass ebenso, wie verschiedene Ausgangssituationen zur gleichen Endsituation führen können, auch ganz unterschiedliche, möglicherweise sogar gegensätzliche therapeutische Ansätze, zum gleichen Endresultat, nämlich einer gesteigerten oder wiederhergestellten Regulationsfähigkeit führen können. Allein schon diese Erkenntnis sollte Grund genug für Toleranz zwischen den verschiedenen Disziplinen der Komplementärmedizin sein. Möglicherweise bewirkt eine Art lokaler Schocktherapie, wie sie die Neuraltherapie darstellt, oder ein subtiler Anstoss, wie bei der Homöopathie, oder eine Veränderung der bewussten Haltung durch Meditation, oder ein radikaler Wechsel des Umfelds durch eine Kur mit romantischer Liebesaffäre am Ende das Gleiche, nämlich eine Rebalancierung des Systems.

Entscheidend scheint mir das Verständnis dafür zu sein, dass die Ebene der Störung und der Therapie eine andere ist als diejenige, die von der konventionellen Therapie angesprochen wird und dass demzufolge auch die therapeutische Leitmetapher eine andere sein muss. Während die Vorreiter der Theoretiker und Forscher innerhalb der konventionellen Forschung langsam erkennen, dass viele Fähigkeiten des Organismus das Resultat einer komplexen systemischen Interaktion eines Netzwerkes darstellen und deswegen nicht oder nur begrenzt von aussen linear-kausal steuerbar sind, haften viele Vertreter der Komplementärmedizin immer noch an einem lokal-linearen Verständnis des Organismus und versuchen mehr oder weniger krampfhaft, sich mit lokalen Theorien anzubiedern oder sich in vage Spekulationen zurückzuziehen. Manche Vertreter versuchen subtil-kausale Prozesse plausibel zu machen, etwa wenn man innerhalb der Homöopathie nach dem «Informationsträger» der Substanz sucht oder wenn man innerhalb der «energetischen Medizin» durch irgendwelche elektromagnetische «Energien» oder «Schwingungen» vermeintliche «Ursachen» von Störungen, die in vermuteten «falschen Frequenzen» oder «Fehlinformationen» zu suchen seien, beheben will. Das Problem bei solchen Pseudotheorien scheint mir zu sein, dass sie nun gerade auf dem gleichen Parkett tanzen wie die Vertreter des Maschinenparadigmas des Organismus, nur vielleicht mit etwas extravaganteren, dafür aber rutschigeren Schuhen. Es scheint mir für die Glaubwürdigkeit der Komplementärmedizin und ihre innere sowie institutionellen Annäherung an den Hauptstrom der Wissenschaft von Bedeutung zu sein, dass sich ihre Vertreter klarmachen, dass sie im wesentlichen komplexe Netzwerkfehler eines sich selbst regulierenden Systems zu beheben versuchen, und zwar mit Massnahmen und Impulsen, deren Wirkweise in den meisten Fällen völlig unklar ist. Ein subtiler Materialismus ist hier nicht hilfreich. $\mathrm{Zu}$ behaupten, wir hätten ja schliesslich auch einen kausalen, lokalen und materiell dingfest zu machenden Mechanismus aufzuweisen, auch wenn er eben subtil ist, etwa in Form von Clusterbildungen, minimalen Strömen, kohären- 
ten Lasern, besonders angepassten Frequenzen, elektromagnetischen Strahlungen in einem subtilen Bereich des Spektrums, rosaroten Mikrowellen oder was sonst alles an theoretischen Behauptungen kursieren mag, scheint mir nicht nur wissenschaftspolitisch töricht, sondern intellektuell unredlich $\mathrm{zu}$ sein. Oftmals stützen sich solche Behauptungen auf wenige Untersuchungen, wenn es überhaupt welche gibt, die in kaum einem Fall unabhängig repliziert sind und bei genauerer Betrachtung so gut wie nie stabil sind. Und selbst wenn materielle Strukturen nachweisbar sind, wie manche noch in Arbeit befindliche Studien zur Strukturaufklärung homöopathischer Potenzen nahezulegen scheinen, ist damit noch lange nicht klar, ob und wenn ja, wie diese mit den therapeutischen Effekten zusammenhängen. Damit soll nicht gesagt sein, dass derartige Untersuchungen Unsinn wären. Im Gegenteil, die materiellen Prozesse aufzuklären, die mit einer Intervention einhergehen, diese ermöglichen oder von ihr ausgelöst werden - Potentialschwankungen der elektrodermalen Aktivität bei Akupunkturpunkten, zentralnervöse Reaktionen nach einer Intervention, Erfassung der elektromagnetischen Sensitivität von Organismen - all dies sind legitime und wichtige Forschungsfragen. Allerdings sollten wir uns hüten, aus anfänglichen Resultaten sofort zum berühmten «Jetzt ist es bewiesen, wir haben's immer gewusst, dass die Therapie xy wissenschaftlich nachweisbar ist" überzugehen.

Angenommen, wir wüssten durch sorgfältige Untersuchungen und viele unabhängige Replikationen: Durch den homöopathischen Herstellungsprozess werden Strukturveränderungen im Wasser, dem Wasser-Alkohol-Gemisch oder im Milchzucker erzeugt, die stabil bleiben und als «Informationsträger» angesehen werden können; angenommen also, wir würden einen lokalen, kausalen Mechanismus kennen, wie homöopathische Arzneimittel ihre offensichtlichen Wirkungen entfalten können. Dann wäre immer noch nicht klar, wie der Organismus diese «Information» verarbeitet, warum eine solche Information, die ja angeblich eine Kopie der einstmals gelösten Substanz ist, andere und um so viel stärkere Wirkungen entfalten sollte als die Substanz selbst. Dann müsste auch plausibel gemacht werden, warum ausgerechnet diese Zielsubstanz, und nicht die erwiesenermassen vorkommenden Verunreinigungen durch die aus den Gläsern gelösten Ionen [7], Wirkung entfaltet. Wenn wir dieses Beispiel konkret weiterverfolgen, so sehen wir, dass durch eine subtil materialistische bzw. kausale Hypothese kein Problem gelöst werden kann, sondern viele neue hinzukommen. Ich denke, dass dies bei anderen Verfahren nicht anders ist.

Was wäre eine Alternative? Zunächst ist es wichtig anzuerkennen, dass genauso viele Wege, wie in die Krankheit hineinführen, auch wieder herausführen. Eine chronische oder komplexe Krankheit, eine Krankheit 2. Ordnung oder ein Netzwerkfehler kann durch sehr viele Interventionen, die den Organismus zu einer eigenen Aktivität anregen oder das System zunächst einmal stören und durcheinanderbringen, geheilt werden. Die Theorien, die benötigt werden, um eine Thera- piemethode fachgerecht anzuwenden, sind nicht notwendigerweise auch korrekte Beschreibungen dessen, was wirklich passiert. Deshalb ist es denkbar, dass all diese Theorien und Handlungsanweisungen nur dazu dienen, Aufmerksamkeit in eine bestimmte Richtung zu lenken, zu kanalisieren oder gar abzulenken, damit das System eine Möglichkeit erhält, sich neu zu ordnen.

Ein Heiler, der mich unlängst besuchte, hat mir seine eigene Theorie und Praxis etwa so geschildert: Heilung geschähe auf einer sehr tiefen Ebene, jener des «nichtlokalen Geistes». Seine Aufgabe sei es, diese Ebene stellvertretend für den Patienten zu erreichen und damit dem Patienten die Möglichkeit zu geben, dass diese sich in ihm manifestieren kann. Auf der Oberfläche führt er dann Dinge aus, die die Aufmerksamkeit des Patienten fesseln, damit genau dieser Prozess geschehen kann. Er legt etwa die Hände auf die Schulter, lässt Musik spielen, in der unverständliche (und nicht unbedingt sinnvolle) Worte einer polynesischen Sprache erklingen, einfach deshalb, weil man davon ausgehen kann, dass die wenigsten Leute diese Sprache verstehen. Sein Tun beschrieb er als aktives Nichtstun, was das eigentlich Wirksame sei. Seit 20 Jahren macht er das nun so, lebt davon und hat Fallvignetten aufzuweisen, die sehr eindrücklich, ja sogar phantastisch sind, wenn man seinen Worten glauben darf. Und selbst wenn man den Selbstpräsentations- und Selbstbetrugsfaktor abzieht, der ja bei jedem Kliniker zu Buche schlägt, der Fälle präsentiert, bleibt immer noch ein respektabler Erfolg übrig.

Wäre es nicht denkbar und realistisch, dass viele Massnahmen der Komplementärmedizin genau hier, nämlich auf der Ebene des Bewusstseins, «des Geistes», ihre Aktivität und Wirksamkeit entfalten? Könnte es nicht sein, dass die meisten Theorien darüber, wie die vermeintlich spezifischen Effekte zustande kommen, im Grunde nur Beschäftigungstherapie für den Therapeuten sind, damit er, bewusst oder unbewusst, das tut, was dem Organismus oder Geist des Patienten zu tun erlaubt ist, was nötig ist zur Gesundung? Wäre es nicht vielleicht an der Zeit, den subtilen Materialismus, dem viele Komplementärmediziner verhaftet sind, mit selbstkritischen Augen zu betrachten? Wäre es nicht nötig, ein Gegengewicht zu setzen zum überwältigenden materialistichen Weltbild, das die moderne Medizin implizit vermittelt?

Damit ist nicht gesagt, dass ein dualistisches Modell der Welt - Geist versus Materie, Leib kontra Seele, Bewusstsein als eigene Substanz gegenüber dem Körper - die Lösung wäre, oder gar ein neo-idealistisches Bild, wie es etwa von Ken Wilber oder anderen Autoren, die von östlicher Philosophie inspiriert sind, propagiert wird. Die Problematik ist komplex und wird weder in diesem Editorial noch im nächsten zu lösen sein. Wir haben an anderer Stelle vorgeschlagen, dass eine Lösung, die den Komplementaritätsgedanken ernst nimmt, vielleicht vorderhand verträglich wäre [6]. Damit ist gemeint, dass man Geist und Materie als zwei komplementäre Seiten einer einzigen zugrundeliegenden Natur betrachtet, die phänomenologisch getrennt, aber ontologisch ursprünglich eins sind, 
aber gleichwohl separate Facetten des Seins wären. Damit wäre immerhin ein simpler und platter Materialismus, wie er von modernen Theorien des Emergentismus propagiert wird oder gar ein eliminativer, der Bewusstsein insgesamt über Bord wirft, überwunden und dennoch der ursprünglich monistische Impuls der Wissenschaft beibehalten.

Wie auch immer eine individuelle oder wissenschaftliche Lösung des Leib-Seele-Problems am Ende aussehen wird, das Bewusstsein wird darin vermutlich eine grössere Rolle spielen, als dies in momentanen Theorien des Geistes der Fall ist. Man beginnt erst langsam zu erkennen, wie Top-down-Prozesse, also solche, die von höheren Regulationszentren ausgehen, etwa vom Bewusstsein, Bottom-up-Prozesse, die von einzelnen materiellen Komponenten des Organismus gesteuert werden, etwa lokale Immunantwort, Stoffwechselprozesse, usw., beeinflussen. In ähnlichem Sinne könnte es sein, dass die mit dem Bewusstsein zusammenhängenden Effekte für die Komplementärmedizin von Bedeutung sind, seien sie klassisch-lokal zu verstehen - etwa durch Aufmunterung, Verstärken der Hoffnung, all jene Prozesse, die man gewöhnlich mit dem hässlichen Wort Plazebo belegt - oder nichtlokal als direkte (Fern-)Wirkung des Bewusstseins auf einen Organismus. Dann wären innerhalb der Komplementärmedizin genau jene Komponenten verborgen, die der konventionellen Wissenschaft im Verständnis von Krankheit und Gesundung (noch) abgehen, und die Komplementärmedizin würde sich und der Wissenschaft einen sehr schlechten Dienst erweisen, wenn sie aus dem Bedürfnis heraus anerkannt zu werden, ihre Stärke verleugnet, um sich anbiedernd dem implizit materialistischen Dogma zu unterwerfen, das derzeit herrscht. Dies ist wiederum nicht misszuverstehen als Appell für eine verschnupfte Distanzierung des Besserwissers, der sich aus Groll darüber, dass er nicht gehört wird, in sein ätherisches Schloss der tieferen Erkenntnis zurückzieht, sondern als Aufruf zu einem konstruktiv kritischen Dialog mit dem Hauptstrom der Wissenschaft. Anerkennung kommt durch zwei Türen: Eine besteht aus harten, sauberen, hieb- und stichfesten Daten; die andere ist Theorie, die die Fundamente erhellt und nicht verrät, und dabei dem Dialogpartner eine Brücke baut zu einem vertieften Selbstverständnis. Wenn die Komplementärmedizin der konventionellen Medizin hilft, sich und ihre Handlungen und Probleme besser zu verstehen - und damit auch die Komplementärmedizin - dann ist der Dialog geglückt und die Integration in den Hauptstrom der Wissenschaft hat begonnen.

H. Walach, Freiburg i. Br.

\section{Literatur}

1 von Bertalanffy L: General System Theory. New York, Braziller, 1968.

2 Hyland ME: The intelligent body. New Sci 2001;32-33.

3 Maturana HR: The organization of the living: A theory of the living organization. Int J Man-Machine Stud 1975;7:313-332.
4 von Uexküll T, Wesiack W: Theorie der Humanmedizin. Grundlagen ärztlichen Denkens und Handelns. München, Urban \& Schwarzenberg, 1988.

5 Varela FJ, Maturana HR, Uribe RB: Autopoiesis: The organization of living systems, its characterization and a model. Biosystems 1974;5:187-196.
6 Walach H, Römer H: Complementarity is a useful concept for consciousness studies. A reminder. Neuroendocrinol Lett 2000;21:221-232.

7 Witt C: Physikalische Untersuchung homöopathischer Hochpotenzen. Essen, KVC, 2000. 